\title{
RD-Optimization of Hierarchical Structured Adaptive Vector Quantization for Video Coding
}

\author{
Marcel Wagner ${ }^{1}$ and Dietmar Saupe ${ }^{2}$ \\ ${ }^{1}$ Universität Freiburg, Am Flughafen 17, 79110 Freiburg, Germany \\ wagner@informatik.uni-freiburg.de \\ ${ }^{2}$ Universität Leipzig, Augustusplatz 10/11, 04109 Leipzig, Germany \\ saupe@ informatik.uni-leipzig.de
}

This poster contains two contributions to very-low-bitrate video coding. First, we show that in contrast to common practice incremental techniques for rate-distortion optimization such as the generalized BFOS algorithm may clearly outperform the standard technique based on Lagrangian multipliers. This is relevant in cases where the computation of RDpoints has a low complexity. An implementation independent performance measure is used for comparison and run-time experiments are provided.

Second, we report on recent progress of our ongoing research evaluating the prospects of adaptive vector quantization (AVQ) [1] for very-low-bitrate video coding. In contrast to conventional state-of-the-art video coding based on entropy coding of motion compensated residual frames in the frequency domain, adaptive vector quantization offers the potential to adapt its codebooks to the changing statistics of image sequences. The basic building blocks of our current AVQ video codec are (1) block-based coding in the wavelet domain where wavelet coefficients correspond to (overlapping) spatial regions, (2) hierarchical organization of the wavelet coefficients using quad-tree structures, (3) three way coding mode decision for each block (block replenishment, product code vector quantization, new VQ block with codebook update), and (4) rigorous rate/distortion optimization for all coding choices (image partition and block coding mode). This video codec does not apply motion compensation, however. A comparison with standard transform coding (H.263) shows that inspite of the improvements of our coder over previously published AVQ video coders $[2,3]$ it still shows a performance gap of about $1 \mathrm{~dB}$ for some test sequences. We conclude that motion compensation is essential also for codecs based on AVQ. First preliminary tests show that AVQ coders that incorporate motion compensation can become competitive with standard transform coding.

\section{References}

[1] J. E. Fowler and S. C. Ahalt. Adaptive vector quantization using generalized threshold replenishment. In Proceedings of the 1997 IEEE Data Compression Conference, 1997.

[2] R. Hamzaoui, D. Saupe, and M. Wagner. Rate-distortion based video coding with adaptive meanremoved vector quantization. In Proc. of the ICIP'98, Chicago, USA, October 1998.

[3] M. Wagner, R. Herz, H. Hartenstein, R. Hamzaoui, and D. Saupe. A video codec based on R/D-optimized adaptive vector quantization. Poster abstract in proc. of DCC' 99 . Full paper available as Technical Report 119, ftp://ftp.informatik.uni-freiburg.de/ documents/reports/report119/ report00119.ps.gz. 